\title{
Changes in body mass index and outcomes after kidney transplantation: a single centre, retrospective, observational study
}

Adam Arshad ( $\sim$ adam.arshad95@outlook.com )

University of Birmingham College of Medical and Dental Sciences https://orcid.org/0000-0002-38703663

James Hodson

Institute of Translational Medicine, Queen Elizabeth Hospital Birmingham

Khalid Khalil

University of Birmingham College of Medical and Dental Sciences

Adnan Sharif

Department of Nephrology and Transplantation, Queen Elizabeth Hospital Birmingham

Research article

Keywords: BMI Change, Kidney Transplantation,Post-transplant outcomes

Posted Date: August 3rd, 2019

DOI: https://doi.org/10.21203/rs.2.12362/v1

License: (9) (1) This work is licensed under a Creative Commons Attribution 4.0 International License. Read Full License 


\section{Abstract}

Background The aim of this study was to describe the changes in body mass index (BMI) after kidney transplantation and assess how this influences long-term outcomes. Methods Data were collected for all kidney transplant recipients between January 2007 and July 2016. Changes in BMI over the posttransplant period were modelled using a generalised estimating equation. The change in BMI from pretransplantation to six months was then calculated for each patient. These were categorised into three groups: stable BMI (a change of $\pm 1.5 \mathrm{~kg} / \mathrm{m} 2$ ), BMI reduction and BMI increase (changes of $>1.5 \mathrm{~kg} / \mathrm{m} 2$ ), between which a range of outcomes were compared. Results Data was available for 1,344 patients, who had a geometric mean pre-transplant BMI of $27.3 \mathrm{~kg} / \mathrm{m} 2$. This declined significantly $(P<0.001)$, to a geometric mean of $25.6 \mathrm{~kg} / \mathrm{m} 2$ one month after transplantation, before increasing and stabilising to pretransplant levels by 36 months (geometric mean $27.2 \mathrm{~kg} / \mathrm{m} 2, \mathrm{P}=0.522$ ). The $\mathrm{n}=882$ patients with BMI measurements at six months, were divided into groups of reduced $(n=303)$, stable $(n=388)$ and increased $(\mathrm{n}=131) \mathrm{BMI}$, relative to pre-transplantation levels. On multivariate analysis, 12-month creatinine levels were significantly higher in the BMI reduction cohort, with adjusted levels of $160.6 \mu \mathrm{mol} / \mathrm{l}$, compared to $135.0 \mu \mathrm{mol} / \mathrm{I}$ in stable BMI. However, no significant associations were detected between six-month BMI change and patient survival, graft survival, incidence of post-transplant diabetes, cancer, or a range of clinical and histological outcomes (all $\mathrm{P}>0.05$ ). Conclusions Our data demonstrates that $\mathrm{BMI}$ significantly reduces in the first month after kidney transplantation, before increasing to pre-transplant levels at 3-5 years. Furthermore, patients with decreasing BMI at six-months have impaired graft function in the longterm. These observations conflict with the existing literature and warrant further investigation.

\section{Introduction}

Post-transplant weight gain is a well-known occurrence in both obese and non-obese transplant recipients (1-4). It has been documented in all forms of organ transplantation (5-7) and several studies have reported an average 5-10 kg increase in weight in the first year after kidney transplantation $(2,4,8-10)$. Weight gain may differ by geographic region, with an average increase in the first year after kidney transplantation of $2.7 \mathrm{~kg}$ documented in France versus $10.3 \mathrm{~kg}$ in America $(2,11)$, and likely reflects the environmental confounders that affect weight gain among different cohorts. Post-transplant weight gain is significant, as it has been associated with inferior graft and patient survival (3) and is thought to contribute to cardio-metabolic risk profiles including hypertension (3), diabetes (3) and dyslipidaemia (12, $13)$ and ultimately increased risk for cardiovascular-related death $(14,15)$. 
The aetiology of weight gain after kidney transplantation is multifactorial and likely influenced by individual, environmental and clinical factors. Immunosuppression, such as corticosteroids, has been shown to stimulate appetite, as well as induce glucose intolerance, hyperlipidaemia, hypertension, and impair vitamin $D$ metabolism $(2,4,16,17)$. However, immunosuppression with steroid-free protocols fails to significantly reduce this risk, with patients still experiencing weight gain $(10,18)$. Additional influences on weight gain may relate to kidney transplant recipients with excellent graft function no longer being limited in their dietary restrictions that are advised to patients with advance kidney disease or failure (19, 20).

While the impact of recipient or donor body mass index (BMI) on post-transplant outcomes has been detailed in the literature $(21,22)$, the degree of 'risk' conferred by increasing or decreasing BMI after kidney transplantation among recipients is poorly understood. Furthermore, the prevailing literature that is present on this topic is outdated (pre-2001) and is published from centres in the United States. Given the changes in transplant care that have occurred in the last decade, and well documented differences in clinical outcomes among kidney transplant recipients between Europe and the United States (23) (including degree of weight gain seen (11)), we believe a contemporary analysis in a European transplant cohort is warranted.

Therefore, the aim of this study was to explore the evolution of weight change after kidney transplantation and its link to clinical outcomes, to help transplant clinicians make evidence-based decisions in relation to weight amongst kidney transplant recipients.

\section{Methods}

\section{Study_population}


Our analysis included all adult patients (aged 18 years of age and older) receiving a living or deceased donor kidney transplant between January 2007 and July 2016 (excluding recipients of multiple organs). We used BMI as our marker of change in weight, as it is the accepted measure for defining anthropometric height/weight characteristics in adults (24).

\section{Outcome Measures}

Our primary outcome measures were patient survival and death-censored graft survival. Secondary outcome measures included clinical outcomes comprising graft function (creatinine levels at 12-months' post-kidney transplantation), incidence of medical complications (e.g. post-transplant diabetes mellitus, cancer, cardiac events, cerebrovascular events, CMV viremia, urology complications, septicaemia and transplant artery stenosis) and histopathological findings. Since the change in BMI was measured over the six-month period post-transplant, follow up for these outcomes commenced at this time. Any patients where an outcome occurred prior to six months were excluded from the analysis of that outcome.

\section{Immunosuppression protocol}

All patients received the same immunosuppression as discussed in the SYMPHONY protocol, while reducing the amount of tacrolimus received (25). Patients received induction immunosuppression with Basiliximab and methylprednisolone. Maintenance immunosupression was Tacrolimus (12-hour trough level 5-8 ng/L), and Mycophenolate mofetil (2g tapering to $1 \mathrm{~g}$ daily at 6 months).

To identify any dysfunction in the transplanted graft, biopsies were taken and scored according to the BANFF criteria (10). Treatment for patients included corticosteroids for acute rejection, with T-cell depletion if there was steroid resistant rejection. Patients with antibody-mediated rejection were treated with plasmapheresis +/- IVIg.

\section{Statistical Analysis}

We first modelled the changing BMI in the post-transplant period using a generalised estimating equation model. The timing of the measurement was set as the independent variable, and an AR(1) correlation 
structure was used to account for the correlations between repeated measures of $\mathrm{BMI}$ on the same patient. Since the BMI measurements followed a skewed distribution, the values were $\log _{10}$-transformed, prior to analysis, in order to normalise the distribution and improve model fit. The coefficients from the resulting model were then anti-logged, and converted into estimated geometric means with 95\% confidence intervals $(95 \% \mathrm{Cl})$.

To assess the effect of weight change on post-transplant outcomes, we first calculated the change in BMI six months after transplant by subtracting the BMI at transplant from the BMI at six months (i.e. negative values represented a reduction in BMI). Patients were then stratified into one of three groups, based on these values: stable BMI (a change of $\pm 1.5 \mathrm{~kg} / \mathrm{m}^{2}$ ), BMI reduction (reduction of $>1.5 \mathrm{~kg} / \mathrm{m}^{2}$ ) and BMI increase (increase of $>1.5 \mathrm{~kg} / \mathrm{m}^{2}$ ). A range of factors were then compared across these three BMI change groups, using Kruskal-Wallis tests for continuous variables, and $\chi 2$ tests for nominal variables.

A range of outcome measures were then compared across the BMI change groups, with Cox regression models for time to event outcomes and binary logistic regression models for dichotomous outcomes. Creatinine levels were found to follow a skewed distribution, and so values were $\log _{10}$-transformed in order to normalise the distribution, before being analysed using general linear models, whilst the numbers of biopsies were compared using a Kruskal-Wallis test. Multivariable analyses were then performed for each outcome, to account for the effect of confounding factors. Prior to this analysis, continuous factors were divided into categories, based on the tertiles of the distribution, in order to improve model fit. All factors were then considered for inclusion in the model, with a backwards stepwise approach used to select those that were independently predictive of patient outcome. Where significant differences between the $\mathrm{BMI}$ groups were detected on multivariable analysis, the adjusted outcomes for each group were calculated. This was achieved by multiplying each coefficient by the proportion of patients the associated category, and evaluating the resulting model for each of the BMI groups. This gave the expected outcome for the "average" patient in the cohort, hence removing the impact of confounding factors.

All analyses were performed using IBM SPSS 22 (IBM Corp. Armonk, NY), with $\mathrm{P}<0.05$ deemed to be indicative of statistical significance throughout. 


\section{Approvals}

This study received institutional approval and was registered as an audit (audit identifier; CARMS-12578). The corresponding author had full access to all data.

\section{Results}

Trends in BMI During the study period, data were available for a total of $\mathrm{N}=1,387$ transplant recipients, of whom $\mathrm{N}=1,344$ (97\%) had pre-transplantation BMI recorded. These patients had a geometric mean pretransplantation BMI of $27.3 \mathrm{~kg} / \mathrm{m} 2$ (95\% Cl: 27.0 - 27.5), which was found to fall significantly by onemonth post-transplantation, to $25.6 \mathrm{~kg} / \mathrm{m} 2$ ( $95 \% \mathrm{Cl}: 25.3$ - 25.9, $\mathrm{P}<0.001)$. The average BMI was then found to increase progressively over the subsequent months (Figure 1, Table 1), before stabilising at approximately the pre-transplantation levels, reaching a geometric mean of $27.2 \mathrm{~kg} / \mathrm{m} 2$ by 36 months post-transplantation ( $95 \% \mathrm{Cl}: 26.8-27.5, \mathrm{P}=0.522$ vs. pre-transplantation). Baseline Demographics by BMI Change A total of $\mathrm{N}=882(59 \%)$ patients had BMI measurements recorded at both pretransplantation, and six months post-transplantation. Of those with missing data, the majority $(\mathrm{N}=417)$ were repatriated to their referring centre post-transplantation, and so did not attend local follow up for $\mathrm{BMI}$ to be recorded. A further $\mathrm{N}=27$ patients lost their graft and $\mathrm{N}=16$ died within six months of transplantation, and so were excluded. Of the remainder, $\mathrm{N}=85$ did not have their $\mathrm{BMI}$ recorded at the sixmonthly appointment, and $\mathrm{N}=30$ had less than six months of follow up. Patients were then divided into groups, based on the change in BMI from pre- to six months post-transplantation. Over this period, $36.9 \%$ $(\mathrm{N}=303)$ saw a reduction in $\mathrm{BMI}$ by more than $1.5 \mathrm{~kg} / \mathrm{m} 2,15.9 \%(\mathrm{~N}=131)$ had in increase in $\mathrm{BMl}$ by more than $1.5 \mathrm{~kg} / \mathrm{m} 2$, with the remaining $47.2 \%$ ( $\mathrm{N}=388$ ) having a stable BMI (i.e. $\pm 1.5 \mathrm{~kg} / \mathrm{m} 2)$. Table 2 compares the demographic and transplant characteristics of the cohort between these three groups. Overall, recipients with a BMI increase were significantly younger $(P<0.001)$ and had a lower $B M I$ at the time of transplantation $(P<0.001)$. In addition, they tended to receive organs from younger donors $(p<0.001)$, were more likely to be receiving kidneys from living donors $(P<0.001)$ and had a reduced rate of delayed graft function (DGF) ( $P=0.032$ ). Recipients with $B M l$ reductions were conversely older and had a higher baseline BMI. They received organs from older donors and were more likely to receive kidneys from deceased donors (all $P<0.001$ ). Both waiting time and cold ischaemia time followed a ' $U$ ' shaped association, being highest in the BMI reduction and increase groups, and lower in the stable BMI group $(P<0.001)$. BMI change and outcomes On univariable analysis (Table 3$)$, the change in BMI six months post-transplantation was not found to be significantly associated with patient survival $(P=0.384)$, graft survival $(P=0.124)$, incidence of post-transplant diabetes mellitus (PTDM) $(P=0.628)$ or risk of cancer development $(P=0.419)$. However, a significant difference in 12-month creatinine was noted $(P<0.001)$, with median levels being higher in the BMI reduction group $(144.0 \mu \mathrm{mol} / \mathrm{l})$, and lower in the BMI increase 
group $(117.5 \mu \mathrm{mol} / \mathrm{I})$. On account of the previously identified baseline differences between the three groups in recipient, donor and transplant related factors, multivariable analyses were performed in order to account for potentially confounding factors. The effect of the BMI change remained non-significant for all outcomes, except for renal function (Table 4), with the previously noted difference in 12-month creatinine levels remaining significant on multivariable analysis $(P<0.001)$. After adjustment for confounding factors, creatinine levels at 12 -months post-transplantation were found to be $19 \%$ higher in patients that had a reduction in $\mathrm{BMI}$, compared to those with stable BMI. Evaluating the model at the midpoint of the confounding factors gave estimated adjusted creatinine levels of $160.6 \mu \mathrm{mol} / \mathrm{l}$ in the BMI reduction group to $135.0 \mu \mathrm{mol} / \mathrm{l}$ in the stable BMI group, and $131.0 \mu \mathrm{mol} / \mathrm{I}$ in $\mathrm{BMI}$ increase group. We further conducted a subsidiary analysis of clinical and histological outcomes, the results of which are presented in Table 5. Overall, in univariable analysis, changes in six-month BMI were not associated with any risk of cardiac or cerebrovascular events, CMV viremia, septicaemia or transplant renal artery stenosis (all $P>0.05$ ). Furthermore, no association between six-month $\mathrm{BMI}$ change and histological rejection was seen (all $P>0.05$ ).

\section{Discussion}

To our knowledge, this is the largest study to analyse the relationship between BMI or weight change and outcomes after kidney transplantation outside of the United States. Interestingly, BMI was not seen to rise significantly post-transplantation amongst our cohort, with a geometric mean $\mathrm{BMI}\left(\mathrm{kg} / \mathrm{m}^{2}\right)$ of $26.6,27.0$ and 27.2 at six-months $(P<0.001)$, one-year $(P=0.005)$ and three years $(P=0.522)$ after transplantation, compared to 27.3 pre-transplantation. After stratifying the cohort by their change in BMI at six months, we noted a higher average one-year creatinine amongst patients with reducing BMI in multivariate analysis $(\mathrm{P}<0.001)$, with an adjusted average of $160.6 \mu \mathrm{mol} / \mathrm{I}$ in the $\mathrm{BMI}$ reduction cohort, compared to $135.0 \mu \mathrm{mol} / \mathrm{I}$ in the reference stable BMI group. However, there was no significant difference in patient survival, death-censored graft survival or any post-transplant medical complications.

Our results add to the conflicting previous studies in the area. While there are several small single-centre studies on this topic area, analysis including large cohorts are lacking. The most recent analysis comes from Switzerland in an assessment of 777 kidney transplant recipients from 2008 - 2013 (26). Overall post-transplant weight was seen to initially drop in the first six months after transplantation by an

average $1.2 \mathrm{~kg}$. Thereafter, post-transplant weight was seen to gradually increase, with a mean $1.2 \mathrm{~kg}$ weight gain at 3 years after transplantation compared to the six-month value. El-Agroudy and colleagues 
performed a similar analysis in 650 kidney transplantation patients between 1990 and 2001 in Mansoura, Egypt (3). In contrast, their study reported a marked increase in body weight in the first six months after kidney transplantation, with a trend for slow increases in subsequent years. Furthermore, this study stratified patients by their six-month BMI into the following cohorts; $<25 \mathrm{~kg} / \mathrm{m}^{2}, 25-30 \mathrm{~kg} / \mathrm{m}^{2}$ and $>30$ $\mathrm{kg} / \mathrm{m}^{2}$. In multivariate analysis, the authors reported a significantly increased risk in graft failure and patient death amongst the obese cohort. In addition, they observed higher incidence of PTDM, hypertension and ischaemic heart disease in this obese cohort. However, there are methodological differences between our analyses such as our focus on temporal change in BMI rather than a specific time point.

Interestingly a recent analysis from Harhay et al. identifies pretransplant weight loss prior to transplant of $\geq 10 \%$ from listing to transplantation was associated with prolonged hospital stay, increased risk of graft loss (aHR: 1.11, 95\% Cl: 1.06 - 1.17, p < 0.001) and mortality (aHR: 1.18, 95\% Cl: $1.11-1.25, \mathrm{p}<0.001$ ) relative to the $<5 \%$ weight loss cohort. While this measures weight loss prior to transplantation, and ours is after transplantation, we note similar findings amongst our cohort.

While kidney transplant recipients are appropriately counselled regarding the long-term risk of weight gain, it could be argued the immediate period after stressful kidney transplantation may contribute to weight loss rather than gain. While corticosteroid exposure (which is at its highest in the early postoperative period) should contribute to increased appetite, this could be countered by gastrointestinal side effects from mycophenolate mofetil such as nausea, vomiting and/or diarrhoea attenuating appetite. Post-operative complications or severe illnesses could also impact upon nutritional status, and lead to weight loss as opposed to gain, but there were no suggestions of more significant complications in the group that dropped their BMI from our data analysis (either within the first six months or thereafter). It has been reported that kidney transplant recipients objectively drop their frailty scores in the immediate few months after kidney transplantation, compared to time of transplantation, beyond rebounding (27), and our observed drop in BMI could be a surrogate measure of this physiological change. However, frailty status is not a routinely checked parameter after kidney transplantation and therefore we do not have the ability to check for any association. 
The importance of our data rests in the lifestyle modification advice we offer our kidney transplant recipients. KDIGO clinical practice guidelines for the care of kidney transplant recipients has the recommendation that; 'We recommend that patients are strongly encouraged to follow a healthy lifestyle, with exercise, proper diet, and weight reduction as needed (28). While this lifestyle intervention advice is relevant long-term, we must ensure adequate advice is offered to prevent BMI loss in recipients early after kidney transplantation where it is not intended (e.g. normal weight at time of transplantation). Patients with chronic kidney disease or dialysis therapy often benefit from renal dietitian input, but this can be lost after kidney transplantation and our study reinforces the value of such allied health professional support in the overall care that is offered to patients after kidney transplantation. This is important in light of our results showing inferior graft function at 12-months after kidney transplantation. Further work is warranted to understand the mechanism behind this and to understand what drives the loss of $\mathrm{BMI}$ for some kidney transplant recipients and how this may relate to inferior graft function

There are several limitations to this analysis that we must consider when interpreting the results. This study was retrospective, single centre in design, and we must acknowledge the limitations this provides. Certainly, there will be covariates and confounders that we cannot adjust for in this analysis that could affect graft function and patient survival. For example, the underlying reason for weight loss after kidney transplantation has not been ascertained and this is of critical importance, as BMI loss out of choice as a lifestyle intervention choice will be different to weight due to critical illness. Furthermore, BMI is not an ideal measure of body mass, and does not differentiate muscle and fat mass. However, it provides us with real-world data that can influence the decision-making process to assess post-transplant body mass changes.

\section{Conclusion}

In summary, we have reported a significant weight reduction amongst kidney transplant recipients in after the months after transplantation, with return to pre-transplantation levels after between three and five years. This should reassure transplant physicians that weight gain after transplantation, which is often 
reported in the literature, may not be as common in some transplant cohorts. Importantly, we report inferior graft function amongst patients with the greatest BMI reduction and greater emphasis on assessing and mitigating this weight loss should be enacted by transplant teams.

\section{Abbreviations}

BMI - Body Mass Index

MMF - Mycophenolate Mofetil

PTDM - Post-transplant Diabetes Mellitus

\section{Declarations}

Ethics approval and consent to participate:

This study received institutional approval at the Queen Elizabeth Hospital, Birmingham and was registered as an audit (audit identifier; CARMS-12578). The corresponding author had full access to all data. Written consent to participate was taken from each patient.

Availability of Data and Materials: The datasets used and/or analysed during the current study are available from the corresponding author on reasonable request.

Consent for publication: The results presented in this paper have not been published previously in whole or part, except in abstract form at the conference 'ERA-EDTA 2019' and 'ATC 2019'.

Acknowledgements: None

Competing interests: None

Funding: Not Applicable 
Authors' contributions: AS designed this study. KK and AS conducted the required data retrieval. AA and $\mathrm{JH}$ conducted analysis of the data and AA and AS wrote the manuscript. All authors reviewed the manuscript. All authors read and approved the final manuscript.

\section{References}

1. Liefeldt L, Budde K. Risk factors for cardiovascular disease in renal transplant recipients and strategies to minimize risk. Transpl Int. 2010;23(12):1191-204.

2. Clunk JM, Lin CY, Curtis JJ. Variables affecting weight gain in renal transplant recipients. Am J Kidney Dis. 2001;38(2):349-53.

3. el-Agroudy AE, Wafa EW, Gheith OE, Shehab el-Dein AB, Ghoneim MA. Weight gain after renal transplantation is a risk factor for patient and graft outcome. Transplantation. 2004;77(9):1381-5.

4. Heinbokel T, Floerchinger B, Schmiderer A, Edtinger K, Liu G, Elkhal A, et al. Obesity and its impact on transplantation and alloimmunity. Transplantation. 2013;96(1):10-6.

5. Williams JJ, Lund LH, LaManca J, Kunavarapu C, Cohen DJ, Heshka S, et al. Excessive weight gain in cardiac transplant recipients. J Heart Lung Transplant. 2006;25(1):36-41.

6. Richardson RA, Garden OJ, Davidson HI. Reduction in energy expenditure after liver transplantation. Nutrition. 2001;17(7-8):585-9.

7. Singer LG, Brazelton TR, Doyle RL, Morris RE, Theodore J, International Lung Transplant Database Study G. Weight gain after lung transplantation. J Heart Lung Transplant. 2003;22(8):894-902.

8. Cashion AK, Sanchez ZV, Cowan PA, Hathaway DK, Lo Costello A, Gaber AO. Changes in weight during the first year after kidney transplantation. Prog Transplant. 2007;17(1):40-7.

9. Patel MG. The effect of dietary intervention on weight gains after renal transplantation. J Ren Nutr. 1998;8(3):137-41.

10. Elster EA, Leeser DB, Morrissette C, Pepek JM, Quiko A, Hale DA, et al. Obesity following kidney transplantation and steroid avoidance immunosuppression. Clin Transplant. 2008;22(3):354-9.

11. Ducloux D, Kazory A, Simula-Faivre D, Chalopin JM. One-year post-transplant weight gain is a risk factor for graft loss. Am J Transplant. 2005;5(12):2922-8.

12. Johnson CP, Gallagher-Lepak S, Zhu YR, Porth C, Kelber S, Roza AM, et al. Factors influencing weight gain after renal transplantation. Transplantation. 1993;56(4):822-7.

13. Nazemian F, Naghibi M. Weight-gain-related factors in renal transplantation. Exp Clin Transplant. 2005;3(1):329-32. 
14. Brattstrom C, Granath F, Edgren G, Smedby KE, Wilczek HE. Overall and cause-specific mortality in transplant recipients with a pretransplantation cancer history. Transplantation. 2013;96(3):297-305.

15. Gill JS, Abichandani R, Kausz AT, Pereira BJ. Mortality after kidney transplant failure: the impact of non-immunologic factors. Kidney Int. 2002;62(5):1875-83.

16. Jurewicz WA, Baboolal K. Obesity and transplantation. Transplantation. 2002;74(5):600-1.

17. van den Ham EC, Kooman JP, Christiaans MH, Leunissen KM, van Hooff JP. Posttransplantation weight gain is predominantly due to an increase in body fat mass. Transplantation. 2000;70(1):2412.

18. van den Ham EC, Kooman JP, Christiaans MH, Nieman FH, van Hooff JP. Weight changes after renal transplantation: a comparison between patients on 5-mg maintenance steroid therapy and those on steroid-free immunosuppressive therapy. Transpl Int. 2003;16(5):300-6.

19. Cashion AK, Hathaway DK, Stanfill A, Thomas F, Ziebarth JD, Cui Y, et al. Pre-transplant predictors of one yr weight gain after kidney transplantation. Clin Transplant. 2014;28(11):1271-8.

20. Moore L GA. Patterns of early weight change after renal transplantation. J Ren Nutr. 1996;6(1):21-5.

21. Arshad A, Hodson J, Chappelow I, Inston NG, Ready AR, Nath J, et al. The impact of donor body mass index on outcomes after deceased kidney transplantation - a national population-cohort study. Transpl Int. 2018.

22. Krishnan N, Higgins R, Short A, Zehnder D, Pitcher D, Hudson A, et al. Kidney Transplantation Significantly Improves Patient and Graft Survival Irrespective of BMl: A Cohort Study. Am J Transplant. 2015;15(9):2378-86.

23. Reboldi G, Verdecchia P, Fiorucci G, Beilin LJ, Eguchi K, Imai Y, et al. Glomerular hyperfiltration is a predictor of adverse cardiovascular outcomes. Kidney Int. 2018;93(1):195-203.

24. Nuttall FQ. Body Mass Index: Obesity, BMI, and Health: A Critical Review. Nutr Today. 2015;50(3):11728.

25. Ekberg H, Tedesco-Silva H, Demirbas A, Vitko S, Nashan B, Gurkan A, et al. Reduced exposure to calcineurin inhibitors in renal transplantation. N Engl J Med. 2007;357(25):2562-75.

26. Beckmann S, Nikolic N, Denhaerynck K, Binet I, Koller M, Boely E, et al. Evolution of body weight parameters up to 3 years after solid organ transplantation: The prospective Swiss Transplant Cohort Study. Clin Transplant. 2017;31(3).

27. McAdams-DeMarco M, Isaacs K, Darko L, Salter ML, Gupta N, King EA, et al. Changes in frailty after kidney transplantation. J Am Geriatr Soc 2015; 63(10): 2152-2157.

28. Kasiske BL, Zeier MG, Chapman JR, Craig JC, Ekberg H, Garvey CA, et al. KDIGO clinical practice guideline for the care of kidney transplant recipients: a summary. Kidney Int 2009;

\section{Tables}

Table 1. Generalised estimating equation model of changes in BMl after kidney transplantation. 


\begin{tabular}{|l|r|c|c|}
\hline Time from Tx & N & $\begin{array}{c}\text { Geometric Mean BMI } \\
(\mathbf{9 5} \% \mathbf{C l})\end{array}$ & P-Value \\
\hline Pre-transplantation & 1344 & $27.3(27.0-27.5)$ & - \\
1 Month & 1149 & $25.6(25.3-25.9)$ & $<0.001$ \\
3 Months & 1078 & $26.3(26.1-26.6)$ & $<0.001$ \\
4 Months & 965 & $26.5(26.2-26.7)$ & $<0.001$ \\
6 Months & 822 & $26.6(26.3-26.8)$ & $<0.001$ \\
9 Months & 790 & $26.9(26.6-27.2)$ & $<0.001$ \\
12 Months & 710 & $27.0(26.7-27.3)$ & 0.005 \\
36 Months & 481 & $27.2(26.8-27.5)$ & 0.522 \\
60 Months & 315 & $26.9(26.5-27.3)$ & $\mathbf{0 . 0 3 7}$ \\
\hline
\end{tabular}

Results are from a generalised estimating equation model, with the timing of the measurement as the independent variable, and an AR(1) correlation structure used to account for correlations between repeated measurements on the same patient. The $\log _{10}$-transformed BMI measurements were set as the dependent variable, and the resulting coefficients were anti-logged, and converted into estimated geometric mean BMls. P-values are in comparison to the pre-transplantation BMI, and Bold P-values are significant at $<0.05$. Tx; Kidney Transplantation, Cl; Confidence Intervals

Table 2: Baseline demographics of the study cohort 


\begin{tabular}{|c|c|c|c|c|c|}
\hline & \multirow{2}{*}{ Valid N } & \multicolumn{3}{|c|}{ Change in BMI Six Months Post-Tx $\left(\mathrm{kg} / \mathrm{m}^{2}\right)$} & \multirow{2}{*}{$\begin{array}{c}- \\
\text { P-value }\end{array}$} \\
\hline & & $\begin{array}{c}<-1.5 \\
\text { Reduction in BMI }\end{array}$ & $\begin{array}{c}-1.5 \text { to }+1.5 \\
\text { Stable BMI }\end{array}$ & $\begin{array}{c}>+1.5 \\
\text { Increase in BMI }\end{array}$ & \\
\hline $\mathrm{N}(\%)$ & 822 & $303(36.9 \%)$ & $388(47.2 \%)$ & $131(15.9 \%)$ & - \\
\hline \multicolumn{6}{|c|}{ Recipient } \\
\hline Age (Years) & 822 & $53(44-61)$ & $48(37-57)$ & $39(31-48)$ & $<0.001$ \\
\hline Gender (Male) & 822 & $175(57.8 \%)$ & $239(61.6 \%)$ & $69(52.7 \%)$ & 0.181 \\
\hline $\mathrm{BMI}\left(\mathrm{kg} / \mathrm{m}^{2}\right)$ & 822 & $28.8(25.7-32.3)$ & $26.5(23.7-29.6)$ & $26.4(22.9-30.8)$ & $<0.001$ \\
\hline Weight $(\mathrm{Kg})$ & 822 & $80.1(69.7-92.9)$ & $75.2(64.8-86.4)$ & $74.5(62.5-90.2)$ & $<0.001$ \\
\hline Ethnicity & 822 & & & & 0.068 \\
\hline White & & $192(63.4 \%)$ & $256(66.0 \%)$ & $84(64.1 \%)$ & \\
\hline Black & & $82(27.1 \%)$ & $26(6.7 \%)$ & $16(12.2 \%)$ & \\
\hline Asian & & $15(5.0 \%)$ & $87(22.4 \%)$ & $29(22.1 \%)$ & \\
\hline Other & & $14(4.6 \%)$ & $19(4.9 \%)$ & $2(1.5 \%)$ & \\
\hline Diabetes & 822 & $39(12.9 \%)$ & $27(7.0 \%)$ & $11(8.4 \%)$ & 0.028 \\
\hline Hypertension & 822 & $157(51.8 \%)$ & $202(52.1 \%)$ & $54(41.2 \%)$ & 0.079 \\
\hline CMV Positive & 822 & $180(59.4 \%)$ & $214(55.2 \%)$ & $83(63.4 \%)$ & 0.214 \\
\hline Dialysis at Transplant & 822 & $232(76.6 \%)$ & $265(68.3 \%)$ & $100(76.3 \%)$ & 0.031 \\
\hline Smoking & 638 & $26(11.8 \%)$ & $35(11.7 \%)$ & $20(16.7 \%)$ & 0.349 \\
\hline \multicolumn{6}{|c|}{ Donor } \\
\hline Age (Years) & 653 & $53(41-61)$ & $48(38-57)$ & $45(32-52)$ & $<0.001$ \\
\hline BMI $\left(\mathrm{kg} / \mathrm{m}^{2}\right)$ & 494 & $25.6(23.4-28.4)$ & $25.8(23.5-28.4)$ & $25.7(23.3-29.2)$ & 0.961 \\
\hline Ethnicity & 508 & & & & 0.660 \\
\hline White & & $145(82.4 \%)$ & $192(82.8 \%)$ & $82(82.0 \%)$ & \\
\hline Black & & $15(8.5 \%)$ & $16(6.9 \%)$ & $11(11.0 \%)$ & \\
\hline Asian & & $12(6.8 \%)$ & $20(8.6 \%)$ & $4(4.0 \%)$ & \\
\hline Other & & $4(2.3 \%)$ & $4(1.7 \%)$ & $3(3.0 \%)$ & \\
\hline \multicolumn{6}{|c|}{ Transplant } \\
\hline Donation & 719 & & & & 0.001 \\
\hline Living & & $99(38.7 \%)$ & $190(55.2 \%)$ & $59(49.6 \%)$ & \\
\hline$D C D$ & & $41(16.0 \%)$ & $33(9.6 \%)$ & $11(9.2 \%)$ & \\
\hline$D B D$ & & $116(45.3 \%)$ & $121(35.2 \%)$ & $49(41.2 \%)$ & \\
\hline Waiting Time (Days) & 822 & $428(109-1330)$ & $368(114-978)$ & $465(154-1337)$ & 0.121 \\
\hline HLA Mismatch & 792 & & & & 0.267 \\
\hline 1 & & $34(11.5 \%)$ & $41(11.0 \%)$ & $10(8.0 \%)$ & \\
\hline 2 & & $68(23.1 \%)$ & $98(26.3 \%)$ & $42(33.6 \%)$ & \\
\hline 3 & & $157(53.2 \%)$ & $176(47.3 \%)$ & $57(45.6 \%)$ & \\
\hline 4 & & $36(12.2 \%)$ & $57(15.3 \%)$ & $16(12.8 \%)$ & \\
\hline ABO-incompatible & 822 & $23(7.6 \%)$ & $30(7.7 \%)$ & $8(6.1 \%)$ & 0.820 \\
\hline DGF & 724 & $51(19.0 \%)$ & $40(11.9 \%)$ & $14(11.7 \%)$ & 0.032 \\
\hline CIT (Minutes) & 739 & $836(377-1151)$ & $602(180-972)$ & $630(180-1080)$ & $<0.001$ \\
\hline
\end{tabular}

Data are reported as median (IQR), with P-values from Kruskal-Wallis tests, or as N's and column percentages, with P-values from $\mathrm{X}^{2}$ tests, as applicable. Bold P-values are significant at P<0.05.CIT, Cold Ischaemia Time; DBD/DCD; Donations after Brainstem/Cardiac Death. 
Table 3. Univariable analysis of patient outcomes by BMI difference

\begin{tabular}{|c|c|c|c|c|c|c|c|}
\hline & \multirow{3}{*}{$\mathbf{N}$} & \multirow{3}{*}{$\begin{array}{l}\text { Overall } \\
\text { P-Value }\end{array}$} & \multicolumn{5}{|c|}{ Change in BMI Six Months Post-Tx $\left(\mathrm{kg} / \mathrm{m}^{2}\right)$} \\
\hline & & & \multicolumn{2}{|c|}{$\begin{array}{c}<-1.5 \\
\text { Reduction in BMI }\end{array}$} & \multirow{2}{*}{$\begin{array}{c}-1.5 \text { to + } 1.5 \\
\text { Stable BMI } \\
\text { Statistic }\end{array}$} & \multicolumn{2}{|c|}{$\begin{array}{c}>+1.5 \\
\text { Increase in BMI }\end{array}$} \\
\hline & & & Statistic & P-value & & Statistic & P-value \\
\hline Death & 821 & 0.384 & $\begin{array}{c}1.56 \\
(0.76-3.21)\end{array}$ & 0.222 & Reference (1.00) & $\begin{array}{c}0.93 \\
(0.33-2.5 \varepsilon\end{array}$ & 0.885 \\
\hline DCGL & 755 & 0.124 & $\begin{array}{c}1.92 \\
(0.99-3.71)\end{array}$ & 0.052 & Reference (1.00) & $\begin{array}{c}1.11 \\
(0.46-2.65\end{array}$ & 0.823 \\
\hline 12-Month Creatinine* & 678 & $<0.001$ & $\begin{array}{c}1.17 * \\
(1.09-1.26)\end{array}$ & $<0.001$ & Reference (1.00) & $\begin{array}{c}0.93^{\star} \\
(0.85-1.02\end{array}$ & 0.129 \\
\hline Post-Tx Diabetes** & 501 & 0.628 & $\begin{array}{c}1.42 \\
(0.65-3.10) \\
\end{array}$ & 0.380 & Reference (1.00) & $\begin{array}{c}1.35 \\
(0.59-3.09 \\
\end{array}$ & 0.475 \\
\hline Cancer & 603 & 0.419 & $\begin{array}{c}1.64 \\
(0.78-3.46)\end{array}$ & 0.194 & Reference (1.00) & $\begin{array}{c}1.37 \\
(0.60-3.13\end{array}$ & 0.455 \\
\hline
\end{tabular}

All analyses were performed using a time-to-event approach using Cox regression models, with results summarised using hazard ratios and 95\% confidence intervals (relative to the Stable BMI group), unless stated otherwise. Bold P-values are significant at P<0.05. DCGL; Death Censored Graft Loss. TX; Transplantation. *Creatinine was found to follow a skewed distribution, and so was $\log _{10}$-transformed, and analysed using a general linear model, with the fold-differences between groups reported. **Patients with pre-Tx diabetes were excluded from the analysis of post-Tx diabetes.

Table 4: Multivariable analysis of factors predictive of 12-month creatinine level 


\begin{tabular}{|c|c|c|}
\hline & Fold Difference (95\% Cl) & P-value \\
\hline Change in BMI Six Months Post-Tx & & $<0.001$ \\
\hline$<-1.5$ (reduction) & $1.19(1.09-1.30)$ & $<0.001$ \\
\hline-1.5 to +1.5 (stable) & Reference (1.00) & - \\
\hline$>+1.5$ (increase) & $0.97(0.88-1.07)$ & 0.563 \\
\hline Recipient Sex & & $<0.001$ \\
\hline Male & Reference (1.00) & - \\
\hline Female & $0.77(0.71-0.83)$ & $<0.001$ \\
\hline Recipient Ethnicity & & $<0.001$ \\
\hline White & Reference (1.00) & - \\
\hline Black & $1.29(1.10-1.52)$ & 0.001 \\
\hline Asian & $0.86(0.78-0.95)$ & 0.002 \\
\hline Other & $1.15(0.94-1.40)$ & 0.179 \\
\hline Donor Age (years) & & $<0.001$ \\
\hline$\leq 40$ & Reference (1.00) & - \\
\hline $41-60$ & $1.19(1.09-1.29)$ & $<0.001$ \\
\hline$>60$ & $1.39(1.23-1.57)$ & $<0.001$ \\
\hline Donor Ethnicity & & 0.029 \\
\hline White & Reference (1.00) & - \\
\hline Black & $1.27(1.07-1.51)$ & 0.006 \\
\hline Asian & $1.12(0.95-1.32)$ & 0.179 \\
\hline Other & $0.94(0.70-1.25)$ & 0.662 \\
\hline Delayed Graft Function & & 0.026 \\
\hline No & Reference (1.00) & - \\
\hline Yes & $1.15(1.02-1.30)$ & 0.026 \\
\hline Donation Type & & 0.004 \\
\hline Living & Reference (1.00) & - \\
\hline$D C D$ & $0.89(0.77-1.04)$ & 0.134 \\
\hline$D B D$ & $1.11(1.02-1.22)$ & 0.021 \\
\hline
\end{tabular}

Creatinine was found to follow a skewed distribution, and so was $\log _{10}$-transformed, and analysed using a general linear model. Variable selection was performed using a backwards stepwise approach, with all factors in Table 1 included at the first step. The coefficients from the resulting parsimonious model were then anti-logged, and converted into fold differences in creatinine for the stated group, relative to the reference. The final model was based on $N=408$, after exclusions due to missing data. Bold $P$-values are significant at $P<0.05$. DCD; Donation after Circulatory Death, DBD; Donation after Brainstem Death.

\section{Table 5: Univariate analysis of post-transplant complications stratified by six-month BMI change}




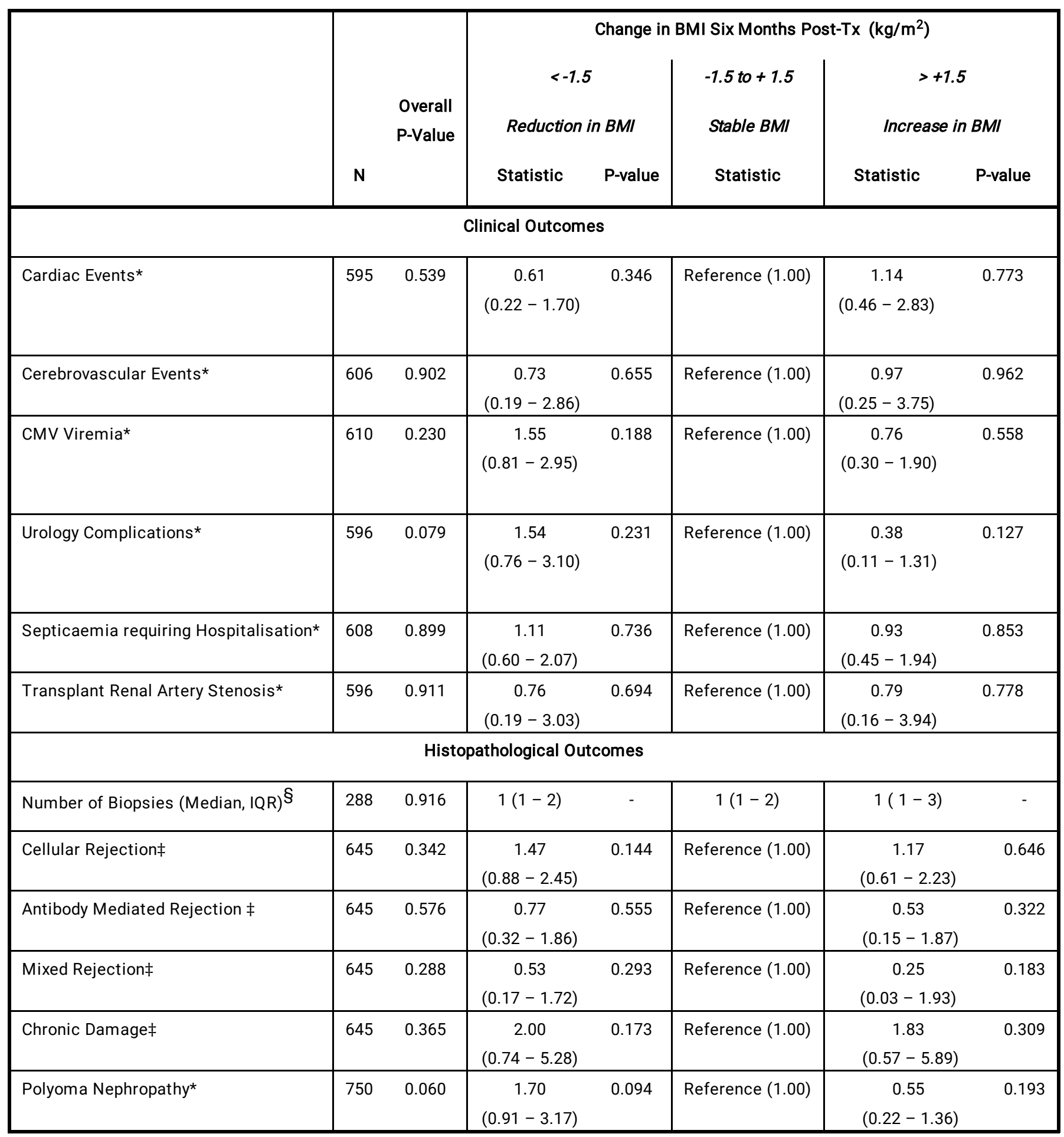

All statistics are relative to the stable BMI group $\left(-1.5 \mathrm{~kg} / \mathrm{m}^{2}\right.$ to $\left.+1.5 \mathrm{~kg} / \mathrm{m}^{2}\right)$. ${ }^{*}$ Time to event data were analysed using Cox regression models, are reported as hazard ratios with 95\% confidence intervals. ${ }^{\ddagger}$ Binary outcomes were analysed by a binary logistic regression model, and the reported statistics are odds ratios with $95 \%$ confidence intervals. \&Continuous outcomes were reported as medians and interquartile ranges, with P-values from a Kruskal Wallis Test. CMV; Cytomegalovirus. Bold Pvalues are significant at $P<0.05$ 
Figures

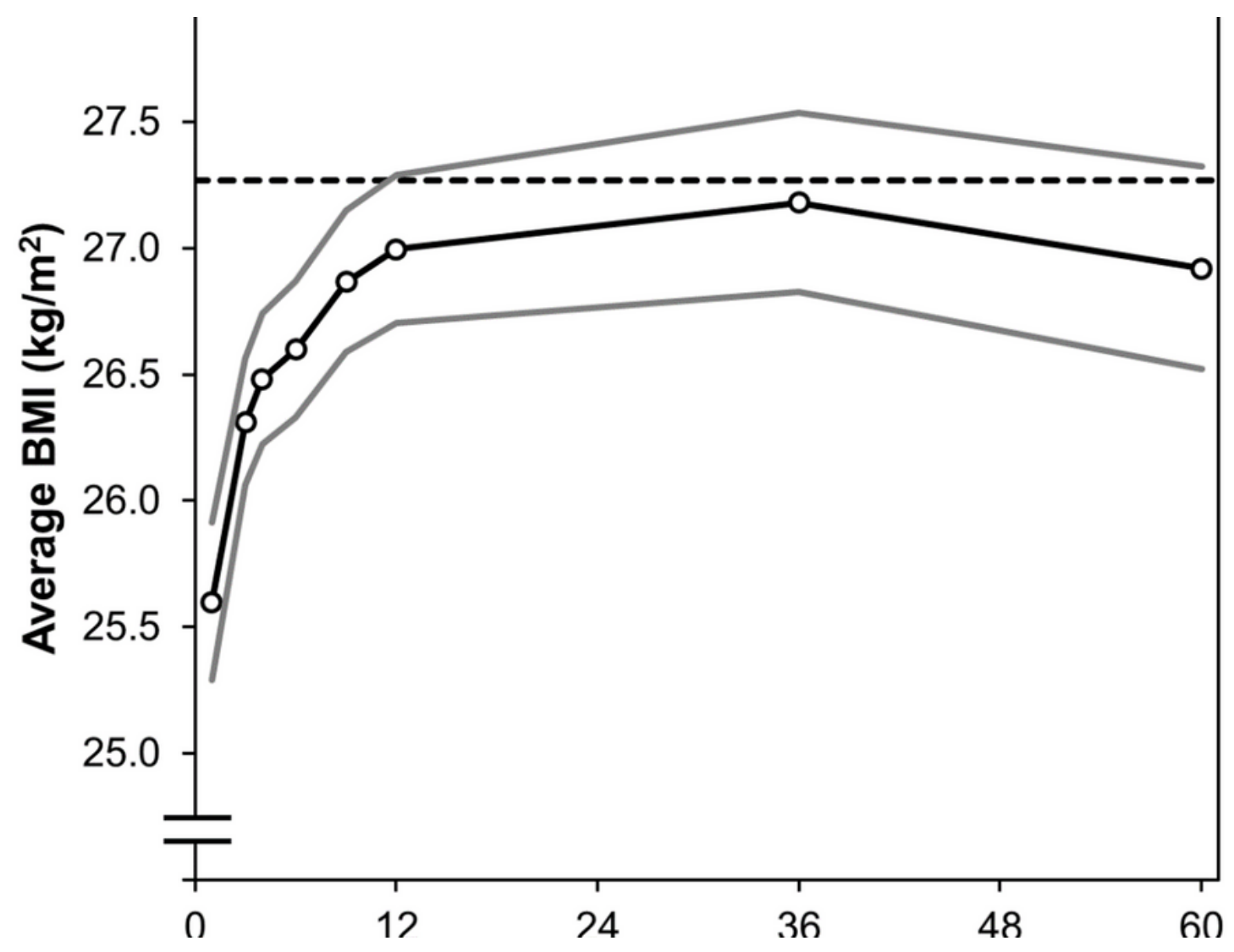

Figure 1

Change in average BMI after kidney transplantation. Average values were calculated from the generalised estimating equation model described in Table 1. The black line represents the average BMI at each point, with the grey lines being $95 \%$ confidence intervals. The broken line is plotted at the average pre-transplant BMI $(27.2$ kg/m2). BMl; Body Mass Index., Tx; Transplantation. 